\title{
Una aproximación a los procesos de subjetivación de las trabajadoras del hogar y el cuidado sindicalizadas ${ }^{1}$
}

\author{
An approach to the processes of subjectivation of domestic and care workers \\ unionized
}

\author{
Karina Fulladosa-Leal ${ }^{2}$ \\ Universidad Autónoma de Barcelona \\ (Rec: mayo 2013 - Acep: junio 2013)
}

\begin{abstract}
Resumen
En los estudios sobre el trabajo de reproducción social se ha enfatizado su carácter de "trabajo invisible". No obstante, las trabajadoras del hogar y del cuidado asalariadas en Cataluña han decidido conformar un sindicato para promover su visibilidad y defender sus derechos. En este artículo me propongo una aproximación a la construcción de subjetividad de las trabajadoras del hogar inmigrantes sindicalizadas, a través de cuatro narrativas realizadas en la ciudad de Barcelona. En la coproducción de estos textos discuto sobre la valoración y significados del trabajo del hogar, la participación de las activistas en el sindicato y las posibles transformaciones que puede implicar estar organizadas. Con ello, se establece una comprensión situada de las trayectorias y los procesos subjetivos de las trabajadoras del hogar y el cuidado articuladas en una acción colectiva como es el sindicato.

Palabras clave: Trabajo doméstico, migración, agencia, género, subjetivación.
\end{abstract}

\begin{abstract}
In studies of the work for social reproduction has been emphasized its character of "invisible work". However, domestic and care workers employed in Catalonia have decided to form a union to promote their visibility and defend their rights. In this article I propose an approach to the construction of subjectivity of migrant household workers unionized, through four narratives held in the city of Barcelona. Through the co-production of these texts I discuss on the assessment and meanings of domestic work, the participation of activists in the union and possible changes that may involve being organized. Thus, is established a situated understanding of trajectories and subjective processes of domestic and care workers, articulated in collective action as is the union.
\end{abstract}

Key words: Household work, Migration, Agency, Gender, Subjectivity.

\footnotetext{
Este artículo se ha construido gracias a muchos diálogos y encuentros. Agradezco en primer lugar a Cynthia, Isabel, Margarita y Ramona por ser parte de este proceso y compartir sus vivencias y conocimientos, al grupo de investigación FIC (Fractalidades en Investigación Crítica) a mis compañeras y compañeros de Máster en Investigación en Psicología Social, a mi tutora Marisela Montenegro, y a mi madre, a quien dedico este trabajo. Esta investigación forma parte de la investigación doctoral de la autora subscrita al Departamento de Psicología Social de la Universidad Autónoma de Barcelona.

2 Correspondencia a: E-mail karinafulladosa@ hotmail.com.
} 


\section{Introducción}

La actual crisis de cuidados en el Estado Español ha configurado un nuevo orden, compuesto por la reasignación de los roles tradicionales de género y los movimientos migratorios, adquiriendo una dimensión global, constituida por la internacionalización de la división del trabajo. Emergen nuevas ciudadanías que respetan pactos que se suponía superados, en tanto ciudadanos/as de segunda categoría o bien ciudadanías directamente excluidas (Eskalera Karakola, 2004) se hacen cargo de los cuidados, creando las condiciones de una nueva esclavitud en el siglo XXI (Gil \& Orozco, 2011).

Ante los diversos conflictos que puede generar este nuevo escenario para el ejercicio de los cuidados, algunas economistas feministas (Carrasco, 2001; Orosco, 2006) plantean un cambio de enfoque, según el cual es necesario romper con falsas dicotomías privado/público, productivo/reproductivo, para lograr una visión integral del mundo. Ello, en tanto se prevé que son posibles nuevas experiencias orientadas a la transformación de roles estereotipados y de las formas de gestionar los cuidados, que conlleven visualizar sociedades organizadas en torno a las necesidades humanas (Orozco, 2006).

Sin embargo, esto es un camino a recorrer, ya que la mercantilización (pública y privada) de los cuidados en la cadena global (Ehrenreich \& Hochschild, 2003; Orozco, 2006; Catarino \& Oso, 2000; Parreñas, 2001) ha producido un cúmulo de situaciones laborales que reproducen y potencian la precariedad. Ello se asocia a la situación de legalidad (con o sin "papeles"), de migración (elegida o impuesta), de una regulación desigual en cuanto derechos laborales con respecto al resto de las(os) trabajadoras(es) y una división sexual del trabajo que discrimina e invisibiliza el trabajo de la mujer (Carrasco, 2006).

Estas diversas situaciones han impulsado al colectivo de trabajadoras del hogar a desarrollar diversas estrategias con el fin de mejorar sus condiciones laborales, optando tanto por estrategias a nivel individual como colectivo. A nivel individual, algunas investigaciones narran múltiples resistencias performativas (Álvarez, 2008) que construyen significado y activan algunas prácticas cotidianas, tales como negarse a usar uniforme, realizar con mayor lentitud el trabajo, o profesionalizar el trabajo para desmarcarlo de una actividad "natural" femenina, la cual no requiere cualificación (Goldsmith, 2007). Las estrategias colectivas, hacen referencia al asociacionismo, como la sindicalización, que se produce en diferentes partes del mundo, atravesadas por las trayectorias de las trabajadoras y su experiencia migratoria (Goldsmith, 2007).

En el Estado Español, concretamente en Barcelona, se ha conformado en diciembre de 2011 el primer sindicato independiente de trabajadoras del hogar y el cuidado, que han dado en llamar Sindihogar/Sindillar. El sindicato se estructura por la transformación de múltiples experiencias asociacionistas, de las cuales las trabajadoras eran parte, que derivan en la construcción de una organización de base sindical. La misma tiene como objetivo reivindicar sus derechos como trabajadoras y denunciar las condiciones de trabajo dentro de la precariedad económica (economía sumergida, bajos salarios, falta de cobertura por desempleo), política (escaso nivel de organización, dificultades para la negociación ante sus empleadores y otros actores sociales) y social (desvalorización e infravaloración de su trabajo, discriminación por raza/etnia, género, sexualidad).

Por todo lo dicho, en este artículo exploro el camino por el cual transitan las trabajadoras narrándose a sí mismas en la conformación del sindicato, en diálogos con otras posiciones, incluidas la de la investigadora y otros textos afines a la temática. En la coproducción de cuatro narrativas, discuto sobre la valoración y los significados del trabajo del hogar, la participación en el sindicato de las activistas y las posibles transformaciones que puede implicar estar organizadas. Con ello, pretendo contribuir a una comprensión situada de las trayectorias y los procesos subjetivos de las trabajadoras del hogar y el cuidado articuladas en una acción colectiva, como es el sindicato.

En los siguientes apartados desarrollo el marco conceptual y la metodología que ha guiado la aproximación a las narrativas de las participantes, para luego introducir la discusión en tres ejes: (i) narraciones en torno al trabajo del hogar y del cuidado, (ii) la participación en el espacio colectivo, responsabilidades, implicaciones y aprendizajes, y (iii) performatividad: hacia una práctica nueva. Para concluir, se presentan algunas reflexiones que surgen como conexiones de los diferentes conocimientos que se han puesto a dialogar, entre las trabajadoras, la investigadora y los textos consultados.

\section{Posibles deslizamientos: del sujeto sujetado al sujeto agente}

Como he mencionado, las experiencias de las trabajadoras del hogar y el cuidado, pueden encontrarse enmarcadas por diferentes desigualdades o 
discriminaciones vividas, como también por estrategias de resistencia que desarrollan para subvertirlas. En este sentido, en tanto personas, podemos narrarnos a nosotras mismas dentro de un contexto socio-histórico que se encuentra atravesado -definido- por marcos normativos, pero a su vez son las diferentes configuraciones que hacemos sobre este mismo contexto, lo que nos permite un campo de lucha para la transformación.

En las narraciones de las activistas ${ }^{3}$ del sindicato establezco un diálogo que permita comprender cómo se piensan, sienten y actúan, con respecto a ellas mismas, los demás y el entorno, haciendo foco en las temáticas del trabajo del hogar y el cuidado, junto a su actividad sindical. Como ha planteado Foucault (1990), en tanto personas interiorizamos una serie de operaciones sobre nosotras mismas y el mundo, dentro de un contexto social que nos precede y condiciona. Pero también habitamos ese mismo espacio de poder, que nos permite negociar posiciones y relaciones. A estos mecanismos Foucault (1990) los llamó las tecnologías del yo, que:

... permiten a los individuos efectuar, por cuenta propia o con la ayuda de otros, cierto número de operaciones sobre su cuerpo y su alma, pensamientos, conducta, o cualquier forma de ser, obteniendo así una transformación de sí mismos con el fin de alcanzar cierto estado de felicidad, pureza, sabiduría o inmortalidad. (p. 48)

En este sentido, podemos considerar que nos encontrarnos en una constante tensión en nuestro proceso de constitución como personas. Esto tiene que ver, por un lado, con (i) los marcos normativos, la sujeción y sometimiento de códigos, leyes que nos preceden, y (ii), por otro lado, con la capacidad de reflexionar sobre nosotras mismas, las(os) otras(os) y el entorno, posibilitando transformaciones sobre nuestras condiciones actuales. De esta manera, esta desviación de la norma se nos presenta como un campo de posibilidades hacia la ruptura de los marcos normativos que nos permiten la rearticulación y resignificación de los contextos anteriores, aunque estos procesos no se den de una forma lineal y constante sino como formas reiteradas de acción.

\footnotetext{
A lo largo del texto se habla de las participantes tanto en su posición de trabajadoras del hogar y el cuidado como en su posición activistas, con esto no pretendemos situar a las personas participantes en unas categorías fijas sino hacer mención a sus diferentes formas de ser, pensar y estar en el mundo.
}

En relación a esta tensión, considero que con el concepto de performatividad (Butler, 2004) facilita una articulación, como posibilidad de un acto de subversión y/o transformación de la norma. Para Judith Butler (2004) como sujetas, somos producto de una reiteración ritualizada bajo ciertas condiciones de prohibición y reglamentación de las normas. Sin embargo, no estamos totalmente determinadas por la misma, es decir que mantenemos una dimensión de incompletud. Esto hace posible la desviación en la repetición y la ruptura de la norma permitiendo la rearticulación y resignificación de los contextos anteriores (Femenías, 2003). A través de las reapropiaciones o resignificaciones, buscamos producir modos de existencia inéditos, como parte de una transformación y modificación de los límites al cual nos encontramos sujetadas. Esto permite significar, reconstruir y resignificar las experiencias, entendiendo que éstas pueden no estar predeterminadas.

La capacidad de acción de las personas está, entonces, caracterizada como práctica de rearticulación o de resignificación inmanente al poder. Es decir, que por un lado las trabajadoras del hogar y el cuidado se encuentran determinadas por posiciones normativas como el género, la clase y la etnia, que establecen relaciones de subordinación y jerarquización en sus relaciones laborales. Pero también existe su capacidad de subvertir las posiciones mencionadas y la posibilidad de producir algún cambio respecto de ellas y su entorno. En este sentido, el tránsito por el sindicato puede generar alguna ruptura de/con los marcos normativos y de esta manera resignificar sus experiencias siendo un campo propicio para pensarse como sujetas políticas.

\section{La metodología: producciones narrativas}

Para el desarrollo de este artículo se realizó cuatro producciones narrativas (Balasch \& Montenegro, 2003) de las cuales surgen cuatro textos coproducidos resultantes de diferentes posiciones entre las participantes, la investigadora y los textos consultados, que permiten comprender distintas visiones y conocimientos que tenemos sobre el trabajo del hogar, el cuidado y la actividad sindical.

La metodología de las producciones narrativas surge en relación con la propuesta epistemológica y política de Haraway (1991) basada en la perspectiva de los conocimientos situados, donde el saber se genera desde unas condiciones semióticas y materiales que dan lugar a una cierta mirada parcial y situada. Las producciones narrativas permiten, entonces, generar 
conexiones parciales, sobre la valoración y significados del trabajo del hogar, la participación sindical y las transformaciones implicadas al organizarse como activistas, entiendo que estas pueden ser unas historias posibles pero no las únicas.

Las narrativas se consideran como parte de las metodologías cualitativas. Entre los antecedentes que hacen mención a este método tenemos las propuestas de Attanapolla (2005), Biglia y Bonet-Martí (2009), Jovchelovitch y Bauer (2005) y Martínez-Guzmán (2009), por nombrar algunas investigadoras que han utilizado esta técnica. Sin embargo, en este recorrido, adhiero a la propuesta de "producciones narrativas" de Balasch y Montenegro (2003) para guiar la construcción de las mismas.

El método de la utilización de narrativas se produce en una serie de sesiones entre la investigadora y las participantes. Allí se comentan los diferentes aspectos del fenómeno en cuestión. Las participantes realizan un relato de su experiencia vivida como parte de ese movimiento, es decir, una "reconstrucción" (Balasch \& Montenegro, 2003). Ello conlleva una textualización de lo dicho, enmarcada por los objetivos que fueron planteados desde el diseño del estudio. Este texto será devuelto a cada una de las participantes para que sea revisado, corregido y/o permita ampliar la visión del fenómeno, que serán introducidas para formar el texto definitivo. "No se recoge, por tanto las palabras de la participante, pero si la forma en que ésta quiere que sea leída su visión del fenómeno" (Balasch \& Montenegro, 2003, p. 45).

La utilización de narrativas se compone por "perspectiva dialógica" (Bajtin, 1979, en Balasch \& Montenegro, 2003), donde el lenguaje es entendido como un proceso relacional activo y abierto, en el cual la persona que habla está localizada en una red de relaciones y géneros de habla. El resultado de las narrativas es un texto híbrido, producto de la coparticipación entre participantes e investigadora (Balasch \& Montenegro, 2003). Por ello, consisten en un campo que propicio para recoger la experiencia de las personas participantes y dar cuenta de sus diversas versiones sobre el mundo: "Las narrativas no buscan probar nada, sino expresar la verdad de un punto de vista, desde una ubicación específica en el espacio y el tiempo" (Jovchelovitch \& Bauer, 2005, p. 72), siendo relevante en el momento de conocer otras subjetividades, donde también se pone en juego la nuestra. Se retoma de este modo, las antiguas tradiciones de la oralidad para generar conocimiento de una forma dialógica (Biglia \& Bonet, 2009). Se producen relatos colectivos que dan cuenta de cómo las personas experimentan eventos, qué sucede a su alrededor y cómo los ven en un momento dado en la historia (Acharya \& Lund, 2002; Lund, 2000; Vandsemb, 1995).

\section{Producciones narrativas, su elaboración y análisis}

El método de la utilización de narrativas se desarrolla a través de una serie de sesiones entre la investigadora y las participantes (Balasch \& Montenegro, 2003). En este estudio, la elaboración de las producciones narrativas se realizó con la participación de cuatro trabajadoras del hogar sindicalizadas. El encuentro se produjo a través de una de las asambleas que realizan todos los sábados, en la sede del sindicato de Sindihogar.

La selección de las participantes se llevó a cabo a través de la presentación de la idea seminal de esta investigación en Sindihogar y optar por realizar las narrativas con las trabajadoras que en aquel momento mostraron su interés en participar. En este sentido, un factor importante fue contar con personas que estuvieran motivadas a ser parte de todo el proceso, ya que las sesiones implican cierta disposición horaria, no asequible a todas las trabajadoras; la ausencia de motivación podría haber generado inconvenientes al momento de fijar y realizar los encuentros.

Una vez presentada la idea y los temas centrales que quería discutir, se acordó la cantidad de sesiones necesarias para elaborar las narrativas. Se convino que las sesiones fueran individuales y que el objetivo de las mismas sería comentar los diferentes aspectos del trabajo del hogar y cuidado, su participación en el sindicato y si el tránsito por el mismo implicaba algún tipo de transformación en sus vidas. A partir de allí se concretaron tres sesiones para realizar un relato de su experiencia, como parte de esa una "reconstrucción" de sus vivencias.

Para la primera sesión estuvo guiada por preguntas abiertas que surgen de la información de los textos consultados, como de algunas ideas previa que se tenía sobre el fenómeno, pero realizadas en formato de diálogo, con el propósito de fomentar la riqueza del fluir conversacional. Este guión no se realizó textual en todas las entrevistas ya que, en virtud de mantener precisamente un diálogo fluido, la emergencia de otros temas de interés tanto de las participantes como de la investigadora, hizo que éste se modificase en el transcurso.

La segunda sesión se concretó luego de realizar la textualización (Balasch \& Montenegro, 2003) de 
la entrevista. En un primer momento se transcribió la conversación y se conformó un texto buscando una coherencia en el diálogo, para que tanto la presentación como su lectura resultase coherente y comprensible.

Este texto se devolvió a cada una de las participantes para su revisión, corrección y para la discusión de las interpretaciones realizadas por parte de las participantes y yo. En este caso, algunas fueron devueltas vía mail, ya que los tiempos de trabajo y situaciones personales de las trabajadoras no hizo viable la reunión presencial.

De esta manera se añadieron las correcciones o modificaciones planteadas, como algunas preguntas aclaratorias sobre los diálogos mantenidos. Por último, en la tercera sesión se introdujo al texto las modificaciones realizadas para formar la producción narrativa definitiva, esto último, en caso que hubiera algo que modificar. En este sentido "no se recoge, por tanto las palabras de la participante, pero sí la forma en que esta quiere que sea leída su visión del fenómeno" (Balasch \& Montenegro, 2003, p. 45).

\section{Presentación de las participantes}

A continuación presento una breve reseña de las participantes para contextualizar las narrativas. Por solicitud explícita de ellas, luego del proceso de consentimiento informado, se da cuenta de sus nombres originales y no se emplean pseudónimos. La información que se brinda no es otra que la que resultó del proceso de construcción de las narrativas.

Isabel hace cinco años que se encuentra en España, primero residió en Madrid y luego en Barcelona. En este momento se encuentra desempleada. Sus primeros trabajos fueron de trabajadora del hogar interna, y el último de asistenta en una residencia.

Margarita llegó a España directamente contratada desde su país para desempeñarse como trabajadora del hogar interna. Luego de una mala experiencia vuelve a Panamá y allí decide nuevamente probar suerte y regresar. Sus últimas labores fueron de trabajadora doméstica externa; actualmente trabaja de forma esporádica en algunas casas.

Ramona desde su llegada ha vivido en Barcelona. Ha trabajado siempre como trabajadora del hogar externa. Su primera experiencia como trabajadora del hogar interna es reciente y la acompaña con trabajos por horas en otras casas en sus días libres.

Cynthia llegó a Ámsterdam, a la casa de su hermana e intentó realizar los trámites para radicarse allí. Ante los problemas administrativos que encuentra para ello, decide instalarse en Barcelona. Una vez aquí trabaja de interna en varios sitios. Actualmente realiza trabajos como externa (por horas) y también lleva adelante tareas en la asociación sin fines de lucro que tiene su hermana.

\section{El tratamiento de las narrativas}

Una vez realizadas las narrativas se discutieron los temas planteados, haciendo conexiones tanto con los textos académicos como los de las participantes, produciéndose una narrativa argumental. Siguiendo a Gadamer (1975) la interpretación no consiste en un producto que se genera iniciando desde el supuesto de igualdad entre investigadora y participante, sino contrariamente se da a partir de la distancia que se plantea entre una y otra.

Las narrativas realizadas no quieren ser una descripción del fenómeno, ni pretenden plasmar una imagen del mismo, sino que se presentan como un material para elaborar interpretaciones conjuntas. De esta manera, el análisis narrativo toma las historias en sí mismas, en tanto pueden considerarse como una performance del narrador: al narrarse se performa o crea la realidad en el recordar y en la reconstrucción del pasado, sea cercano o lejano (Riessman, 2002).

El procedimiento seguido es una difracción del fenómeno, es decir, leer las narrativas, identificar en los textos qué valores, significados y percepciones se ponen en juego, mapear las tensiones que se desprendan de los mismos. Así se contrasta el análisis realizado con las participantes, se identifican diferencias, se aclararan algunas temáticas y añaden los comentarios realizados. De este modo, lo que se pretende es la expresión del efecto, que surge de tomar contacto con el objeto a investigar a partir de una posición inicial de la investigadora (Balasch \& Montenegro, 2003).

\section{Resultados}

\section{Posibles desplazamientos: del cuidado de otros al cuidado de sí}

En los siguientes apartados discuto conjuntamente con las participantes y los demás textos consultados las diferentes subjetividades que se despliegan, tanto en relación al trabajo del hogar como en la actividad sindical que llevan adelante. 
El primer apartado se refiere a los diferentes significados del trabajo del hogar y el cuidado delimitados en las prácticas de sí, dentro de los marcos normativos, configurando un modo de "ser trabajadora" (invisible, afectuosa, cuidadora). El segundo, da cuenta de las formas de participación en el sindicato. Por último se discute los posibles desplazamientos que las participantes han realizado a partir de sus vivencias y resignificaciones atravesadas por la actividad sindical.

\section{(i) Narraciones en torno al trabajo del hogar y del cuidado}

La primer temática que surge con las participantes trata de la invisibilidad y negación del valor social que tienen los trabajos de reproducción social.

Las narrativas de las participantes coinciden en que el trabajo del hogar tiene muy poco valor social y mencionan algunas de las razones por las cuales, para ellas, se produce este fenómeno.

En primer lugar, porque a nivel social el trabajo del hogar se encuentra muy desvalorizado, sobre todo en estos momentos que la gente se ampara mucho en el tema de la crisis para no pagar lo que corresponde a las trabajadoras... (Margarita, 2012)

\section{En segundo lugar:}

Se encuentra invisibilizado, no se valora. Las personas mayores que solicitan ayuda, una asistente, piensan que no es trabajo, que tú no haces nada, ya que las labores de casa nunca se han visto valoradas. Ni siquiera como madres, se nos ha valorado. Al decir de mi hermana, hay una "mano invisible" que va limpiando, que pasa la fregona, y los demás no se dan cuenta. (Cynthia, 2012, p. 3)

En estas narrativas se plantean puntos de conexión respecto a las dificultades de conceptualizar, medir y valorar el trabajo de cuidados, y en particular cómo se produce esta dimensión subjetiva en torno a la invisibilidad (Carrasco, 2006). Es decir, el trabajo de cuidado no sólo se refiere a una actividad física, sino que se encuentra relacionado con actividades que no son cuantificables, en tanto son productoras y generadoras de afecto.

Siguiendo las palabras de Carrasco (1991) esta desvalorización se encuentra asociada a los escasos lugares donde se cuestiona su importancia para la sostenibilidad de la vida y, precisamente como señala Margarita (2012), donde se desdibujan muchas responsabilidades. Podemos decir entonces que esta invisibilidad "es una estrategia de subordinación y no un reflejo de su poco peso relativo" (Juliano, 2001, p. 18) para la reproducción de la vida.

El ocultamiento de una tarea de valor, como lo es la sostenibilidad de la vida, no se presenta de forma inocente (Carrasco, 2001), sino que está relacionada con un conflicto de intereses, donde los trabajos y actividades que forman parte de los procesos de reproducción no gozan de las mismas valoraciones, resultado de una tradición patriarcal liberal.

La narrativa de Isabel introduce otro tema que está vinculado a la falta de valor social, pero que ella adjudica a una pauta de género que está relacionada con el trabajo del hogar.

El rol del hombre al de la mujer es distinto, también en cuanto a la limpieza. A los hombres se les ha enseñado de niño, que solo estaban para jugar, y las niñas tenían que limpiar la casa. Las abuelas no permitían que los niños entraran a la cocina y sin embargo las niñas si tenían que ayudar. Esto viene de siglos, se ha sobreexplotado a la mujer en este sentido, el hecho de ser mujer nos ha condicionado mucho para hacer cosas, por ejemplo ni plantearte poder estudiar muchas veces (Isabel, 2012, p. 3).

Podemos decir que la división sexual del trabajo trae aparejado un modo de producción y de subjetivación que sigue pautas de género (Izquierdo, 2004), donde la "mujer" se subjetiva como cuidadora, en el entorno privado/doméstico y con disposición a satisfacer las necesidades ajenas.

Respecto a esto Lagarde (1996) señala, que la normativa de la sexualidad produce "múltiples mecanismos pedagógicos, coercitivos, correctivos, que a su vez son mecanismos de poder de dominio que aseguran mayores posibilidades de desarrollo a algunos sujetos de género frente a otros que, por su género y su situación vital, tienen reducidas oportunidades" (p. 29).

Estos roles adjudicados se presentan como una forma de internalizar una forma de ser y estar para otros, que en palabras de Ramona (2012) está vinculado a considerar su trabajo como gratificante, al cuidar a personas mayores, lo que para ella significa un aprendizaje de amor y respeto hacia aquella persona que no puede hacerlo por sí misma.

Esta es una de las posibles posiciones que se presentan cuando se dialogan sobre los modos de reproducción de ser "cuidadora", y que tienen que ver con los procesos de significación, configurados en lo que es "ser para otros" (Lagarde, 1997), adscribiéndose 
de esta manera a las identidades femeninas, donde los cuidados no son socializados y sí naturalizados. De alguna manera, esta naturalización continúa ubicado a la "mujer" en el rol tradicional, haciéndola cargo de la reproducción social.

La naturalización de esta tarea, dentro del orden de lo "exclusivamente" femenino, ha desencadenado una visión que la relaciona con un trabajo de poco prestigio, silenciosa, invisibilizada y sin reconocimiento social. Ello deriva en una amplia tradición de discriminación del trabajo de las mujeres en distintas partes del mundo (Contreras, 2006).

Una vez que estas tareas se trasladan al mercado (como forma de la externalización y mercantilización del trabajo del hogar y el cuidado) reproducen un imaginario negativo y ello es a la vez causa y consecuencia de su fuerte feminización (Parella, 2002). Esta cadena de significantes, se desplaza hacia las personas que trabajan en el hogar y el cuidado de forma remunerada, desplegando unas consecuencias de orden jurídico, económico, social y cultural (Lerussi, 2008).

Otro tema emergente en los diálogos con las participantes hace referencia a las diversas experiencias en torno al trabajo realizado como internas y externas, donde se presentan diferentes narrativas y formas de pensarse. En estas narrativas se marcan fuertes relaciones de subordinación y jerarquización que encuentran en su trabajo, sobre todo en el que hacen "puertas adentro".

Con respecto al trabajo de interna Margarita nos señala que:

... estar tanto tiempo encerrado, sin tener la opción de compartir tus ideas, hablar con otras personas, llega un momento que te perjudica psicológicamente. No sé si a otros les sucede lo mismo, pero yo necesito hacer cosas que obliguen a mi mente a estar trabajando constantemente, sino siento que se me va reduciendo mi capacidad intelectual (Margarita, 2012, p. 1).

Estas condiciones de trabajo, conllevan vestigios heredados de las relaciones serviles precapitalistas entre el empleador(a) y la persona empleada (Larrañaga \& Jubeto, 2009). Las palabras de Margarita muestran cómo se produce una enajenación de la persona y como los espacios vitales y de trabajo se ven desdibujados, debido a la convivencia con las(os) empleadoras(es). Esto, además de encontrarse reducidos los espacios de privacidad, produciéndose subjetividades que tienden a enmarcarse dentro de la falta de capacidad, autonomía e identidad (Margarita, 2012).

Otra forma de narrar las relaciones de jerarquización y subordinación marcada por una diferenciación de clase, empleada/empleadora se menciona cuando:

...una empleadora te manda a la farmacia o algún sito y te dice que tienes ir uniformada, esto es una manera de denigrar, de mostrar a todo el mundo que esta es mi «chacha», mi «empleada»... Lamentablemente los empleadores y que la gran mayoría son empleadoras mujeres como nosotras... cuando estamos a su servicio nos denigran, nos tratan inferiores a ellas, nos ven inferior a ellas... (Margarita, 2012, p. 4)

Aquí Margarita nos apunta la connotación que tienen los términos "mi chacha", "mi empleada", que implican vestigios de servilismo y sujeción entroncados en la relación laboral. Este relato, deja desprender que las relaciones de clase se encuentran enmarcadas dentro del trabajo del hogar, delimitando la subordinación de las mujeres en términos de relaciones capitalistas de clase así como intra/intergénero (Lagarde, 1996).

La falta de formalidad en la relación laboral (Isabel, 2012; Margarita, 2012; Cynthia, 2012) y la arbitrariedad respeto a las condiciones de trabajo, acarrea también determinadas relaciones de subordinación y dominación. Sobre todo teniendo en cuenta que existe una relación individual y directa entre la trabajadora y empleadora, desprotegida de un marco institucional y con escaso poder social de negociación, tanto individual como colectivo (Castello, 2008). "Para que sea un trabajo reconocido hay que generar conciencia a los empleadores para inscribir a las trabajadoras en la seguridad social" (Ramona, 2012, p.3).

Por otro lado, las narrativas sobre el trabajo del hogar realizado en forma externa, hacen hincapié en una amplitud de los espacios de libertad tanto respecto del tiempo como del lugar de trabajo. El trabajo como externa, es diferente, cambia porque estás en la casa durante un par de horas y luego te vas (Margarita, 2012). "Mi experiencia en este tipo de trabajo es que tú comienzas a ver que aunque no ganes demasiado dinero, haces tu trabajo y te vas, esto es una libertad. Aquí aprendes a valorar tu tiempo" (Cynthia, 2012, p. 2).

El traspaso de un trabajo a otro (interna/externa), da cuenta del resituar el valor de su tiempo y el poder utilizarlo en otras actividades, como participar en el 
sindicato, formarse, contar con tiempo para las relaciones familiares y de ocio. Este tipo de trabajo, significa para las participantes poder ampliar sus márgenes de decisión en relación a su vida y disposición del tiempo, y es una opción preferible, aunque esto pueda repercutir en obtener un menor ingreso económico. Es decir, que hay un cambio de prioridades, donde la disposición temporal cobra mayor importancia que la material.

Siguiendo la narrativa de Cynthia, se menciona un mayor grado de independencia, al culminar el horario laboral: "Este tiempo es importante porque en estos ratos tú puedes hacer cursos, formarte, no hay como tener tu tiempo. Por ejemplo, ahora tengo tiempo para ir a la asociación y al sindicato, ahora puedo participar de estos espacios" (Cynthia, 2012, p. 2).

Este tipo de trabajo implica de cierto modo, abandonar el aislamiento y el encierro que se da en el caso del trabajo como internas, al encontrarse con horarios poco flexibles y restrictivos. Es un paso hacia una disponibilidad mayor de tiempo libre, que repercute en una mayor participación social y política. Se van produciendo otros agenciamientos que tienen que ver con "hacer su vida" recuperando la circulación por otros espacios de la vida cotidiana, que no son los meramente laborales.

En los relatos se van produciendo diferentes subjetividades respecto a sus situaciones laborales, como también a sus primeras experiencias de trabajo y experiencias migratorias que hacen referencia a diferentes estrategias de acomodación en la sociedad de destino. Se generan así procesos heterogéneos, que se inscriben en y en los cuales participan sus propias biografías.

\section{(ii) La participación en el espacio colectivo, responsabilidades, implicaciones y aprendizajes}

La siguiente pregunta que se abre hace referencia a la trayectoria de las participantes en el sindicato y sus diferentes visiones respecto a ser parte del mismo. Es decir, cómo se vincularon al sindicato, que significado ha tenido estar involucradas a este y cómo se articulan los procesos subjetivos singulares en un espacio común.

Las participantes se han implicado en el sindicato a partir de sus experiencias previas en otros espacios asociativos. El organizarse colectivamente se narra como la posibilidad de conocer otras experiencias, formarse y generar acciones sobre sus condiciones concretas como trabajadoras del hogar y el cuidado.
La relación comenzó a través de un proyecto de unas mujeres colombianas. Allí empecé con los talleres de informática y con los talleres de derecho y formación laboral... Un día me han propuesto si quería representar a Maloka ${ }^{4}$ en el sindicato (Ramona, 2012, p. 3-4)... llegué a través de otras asociaciones. En estas reuniones compartimos nuestras historias y experiencias, pero sentía que quedaban ahí sin poder hacer nada... (Margarita, 2012, p. 5). Mi relación con el sindicato comenzó por estar participando en otros espacios asociativos y enterarme de una reunión que se iba a llevar a cabo sobre las trabajadoras del hogar (Cynthia, 2012, p. 4).

Estas narraciones nos hacen pensar el espacio colectivo, como un ámbito propicio para el intercambio de información, generar pertenencia y posibilitar la creación de proyectos en base a las necesidades de las personas.

La asociación representa el espacio en el que se destruye aquel aislamiento social que conlleva vivir en las coordenadas clásicas de la inmigración. Constituye el espacio en el que se permite fomentar y potenciar la solidaridad, y es donde los diferentes colectivos hallan los referentes válidos para encontrarse (Sipi, 2000, p. 358).

Podemos decir que no es azaroso que las participantes se cuestionen la necesidad de formar un sindicato producto de las reflexiones y reuniones en los espacios asociativos, sino que efectivamente estos lugares permiten compartir y solidarizar fuerzas, sobre todo en los primeros momentos de acomodación a una nueva sociedad, leyes y normativas. Estos encuentros previos hicieron posible que las diferentes trayectorias de las trabajadoras se conjugaran y dieran un salto desde las múltiples experiencias asociacionistas, de las cuales eran parte, hacia la construcción de una organización de base sindical y su constitución como activistas políticas.

Por otro lado, las diferentes narrativas señalan que la participación es una forma de compartir experiencias con otras compañeras y conocer otras culturas; es decir: "tratar con diferentes compañeras inmigrantes, con diferentes culturas, te enriquece" (Isabel, 2012, p. 4). "A nivel de grupos y relaciones sociales

\footnotetext{
4 El Colectivo Maloka-Colombia es una asociación sin fines de lucro que se conforma en el año 2002 como un espacio de encuentro entre colombianas/os residentes en Barcelona y catalanas/es sensibilizados por la situación colombiana.
} 
te permite interactuar con otras personas, hacerte retos...” (Margarita, 2012, pp. 5-6). “... [C]omienzas a ver cuáles son tus derechos, a conocerlos y comenzar a preguntarnos cuáles son las necesidades que tenemos como sindicato. Este espacio nos permite relacionarnos y compartir, salir muchas veces de la soledad donde trabajamos" (Cynthia, 2012, p. 4).

El "re-pensarse" dentro del espacio colectivo puede dar lugar a efectuar cierto tipo de operaciones vinculadas con la participación, donde se van generando nuevas estrategias, que, de una manera $\mathrm{u}$ otra, ensanchan los márgenes de participación social y política. "Te permite acercarte a las necesidades que vamos teniendo, a formarnos, a conocer los derechos que tenemos. Aquí sabemos que estamos todas en la misma y esto te permite enfrentar tu trabajo de otra manera" (Cynthia, 2012, p. 5).

La acción colectiva repercute en otras formas de tramitar sus experiencias, tanto como trabajadoras como activistas. Se genera un viraje de lo privado hacia lo público, donde se presenta una narrativa del "nosotras", pensando en actividades que tienen que ver con sus necesidades, más que con las necesidades de un "otro". Se presenta la posibilidad de intercambiar experiencias, que no sólo tienen que ver con las dificultades que se les presentan, sino también con la posibilidad de crear estrategias de resistencias para reivindicar mejores condiciones de trabajo. Por ende, esta puede ser una forma de ampliar el campo de posibilidades (Foucault, 1988), siendo la libertad una de ellas, que se torna más visible en la reconstrucción de experiencias de unas y otras.

En la trama de relatos existen asociaciones vinculadas a una mayor autonomía y capacidad para la acción. Por lo cual, es importante hacer visibles las estrategias no solo de sometimiento sino también de resistencia, siempre concretas y parciales, ya que esto es una práctica que enriquece modos situados de lucha contra dominaciones y sometimientos específicos (Amigot, 2005), permitiendo desafiar la invisibilidad a la que muchas veces se enfrentan las trabajadoras del hogar.

Para finalizar, dialogamos con las participantes respecto de las tensiones que surgen al habitar el sindicato y las alianzas que llevan a cabo para continuar trabajando hacia un objetivo común.

Para Ramona, su participación tiene dos dimensiones: por un lado, el considerar importante participar del sindicato y, por otro, la responsabilidad que implica el desempeñar una actividad sindical, sumándose las diferencias que surgen al tener distintas visiones de los problemas que enfrentan:
Creo que no me he entregado como debía de ser, aunque asumo la responsabilidad de mis tareas, creo que no me he dejado llevar. Siento que es importante participar, aunque para mí se estaba convirtiendo en una manera impositiva de conocer más derechos y leyes que en este momento no puedo asumir. (Ramona, 2012, p. 4)

Existen desafíos que se presentan al tener que conciliar la vida social, sindical y laboral, generando subjetividades cargadas de nuevas obligaciones. De alguna manera se resalta la complejidad de negociar entre el tiempo libre, las actividades fuera del ámbito laboral (como las tareas domésticas y cuidados de sus propios hogares, el descanso y el ocio) y las del sindicato.

Con respecto a las dificultades del trabajo en conjunto dentro del sindicato, se menciona su carácter procesual, en tanto son parte de los nuevos aprendizajes y la posibilidad de trabajarlos y dialogarlos conjuntamente.

El trabajo en colectivo nos permite aprender a conocernos, a relacionarnos, aceptarnos como somos cada una. Tomar decisiones en conjunto, aunque sean temas de jaleo, sabiendo que a veces soy un poco autoritaria, pero son cosas que tenemos que aprender y aceptarnos entre todas. Sé que no es fácil, pero para trabajar juntas debemos tomarlos como parte de nuestros aprendizajes (Margarita, 2012 , p. 5)... hay que ir trabajando, juntar fuerzas y pedir ayuda para que nos asesoren. Saber cuáles son nuestras debilidades y fortalecerlas, para atraer a más personas (Cynthia, 2012, p. 5). Pienso que es una tarea compleja, porque cuando somos muchas es difícil ponerlos de acuerdo. Pero como digo siempre rescato de lo negativo lo positivo. (Isabel, 2012, p. 4)

En estas narrativas se nos presentan diferentes subjetividades dentro de la acción colectiva, en tanto sus experiencias singulares están marcadas por diferentes procedencias, culturas, saberes, costumbres. Por lo tanto, las trabajadoras tienen que conciliar experiencias, vivencias y contradicciones como mujeres, trabajadoras y activistas, donde la construcción de un espacio común será producto del encuentro-desencuentro de identidades colectivas múltiples y no coherentes entre sí (Biglia, 2005).

Es decir, que las diferencias en los colectivos minorizados no deben cristalizar el énfasis por una construcción identitaria unitaria, sino generar la posibilidad 
de una estricta cooperación para la subversión de las opresiones (Biglia, 2005). De esta manera, se trata de evitar caer en definir una subjetividad homogénea de todo el colectivo; al contrario, a partir del reconocimiento de las diferencias y semejanzas, es posible plantear trabajar hacia un objetivo común.

Por último, con respecto a los diferentes grados de implicación de las participantes en el sindicato, se señala que la participación social y política, particularmente de las activistas de Sindihogar, se produce de forma desigual y discontinua, y si bien significa aprendizaje, obtención de recursos y empoderamiento, no logra ser constante y cotidiana (Lagarde, 1996). Esto se debe a la necesidad de conciliar las tareas de reproducción social y familiares, donde de forma situada el tipo de trabajo que desempeñan, el constreñimiento de sus horarios y la necesidad de trabajar en múltiples casas, conspira contra una participación y apropiación del espacio colectivo de forma más constante y activa.

\section{(iii) Performatividad: hacia una práctica nueva}

En estos párrafos se despliegan los relatos que hemos mantenido con las participantes en torno a posibles desplazamientos, es decir, pequeña acciones que tengan nuevos significados sobre sus experiencias.

La decisión de conformar el sindicato surge como la instancia de performance de nuevos espacios y prácticas. Es decir, no sólo reunirse para contar sus experiencias era algo necesario, sino el poder hacer algo respecto de ellas; se trata de algo significativo. Tal como hace referencia Margarita, (2012):

En estas reuniones compartimos nuestras historias y experiencias, pero sentía que quedaban ahí, sin poder hacer nada, porque las asociaciones no tienen los mecanismos para hacer frente a esto. Es decir, que digan la compañera está pasando por esta situación, vamos a entablar una acción... Así llega el momento, surge la inquietud de que se forme un sindicato de trabajadoras del hogar (2012, p. 4).

De esta forma, Margarita relata la necesidad de pasar del discurso de diferentes experiencias narradas por sus compañeras y compartidas en un espacio común (las asociaciones), a la acción concreta de poder hacer algo con esos malestares. Por lo cual este "momento" puede ser comprendido como ritual que inaugura una "historia condensada" (Butler, 2004), que surge en la inquietud de muchas trabajadoras del hogar a partir de muchas reuniones.

Podemos decir, que este tipo de acciones se acercan a una ruptura con lo estático y cristalizado, de una manera de estar en el mundo como trabajadoras hacia la iterabilidad de la acción, que se presenta como fuerza y posibilidad de un acontecimiento (Butler, 2004) al constituirse como activistas. Es decir, pensar que es posible que se dé algo novedoso al resignificar el signo, donde las participantes al contar sus experiencias van transformando sus historias. Ello produce no sólo que estas historias entren en diálogo, sino que colectivamente puedan hacer algo con ellas, actuarlas de otra manera.

En el espacio sindical, se van reiterando cierto tipo de acciones, como son las manifestaciones y concentraciones realizadas, las entrevistas en los medios de comunicación, donde las participantes ocupan otros lugares y tienen otros interlocutores. El organizarse por ellas mismas, al ser un sindicato independiente, llama mucho la atención y gracias a ello han logrado un impacto en la prensa y en los medios (Cynthia, 2012).

Nos llaman para pedirnos entrevistas y esto te hace ver las cosas de otra manera, porque también incentiva a que otras se acerquen (Cynthia, 2012, p. 5). Es un reto lograr este cambio, por eso es importante, es la gratificación de estar lejos de mi familia y de otras muchas situaciones. (Margarita, 2012, p. 5)

El sindicato es la posibilidad de conformar determinadas acciones públicas y políticas, que va construyendo nuevas posiciones de sujetas en tanto activistas. Concretamente Cynthia lo vivió a través de una experiencia de trabajo, donde luego de estar involucrada en el sindicato, exigió ante sus empleadores todas las condiciones legales de trabajo y aunque la respuesta fue negativa, consideró que fue un triunfo reclamar lo que es justo como parte de sus derechos laborales (Cynthia, 2012).

Luego que las participantes son interpeladas a actuar de acuerdo a unos marcos normativos, se suscita un desplazamiento de sus posiciones, tal como lo explica Cynthia (2012) al poder exigir bajo qué condiciones laborales está dispuesta a trabajar. Entendemos que son fenómenos complejos y que las diferentes acciones que emerjan tendrán que ver con "la experiencia, en proceso, que se actualiza a través de actos performativos que se reproducen y, por tanto, posibilitan la transformación de los marcos normativos en los que se 
inscribe" (Montenegro, Galaz, Yufra \& Montenegro, 2011). Es un proceso, que como narra Cynthia (2012) se va gestando a través de las actividades que realizan, en base a las necesidades que se les presentan.

Las múltiples resistencias performan las narrativas que construyen significado y activan un presente de inciertas y precarizadas alianzas, que oscilan según el momento y las demandas, activando con el mismo movimiento un crisol de resistencias cotidianas, donde las manifestaciones no explícitas o abiertas, se traducen en formas sutiles de resistencias, frecuentemente acompañadas de un cumplimiento estricto de un rol o una función social. (Álvarez, 2008, p. 43)

Por último señalar que estas narrativas, también se presentan como acciones que constituyen, median y transforman la realidad -performan-, en tanto se van escribiendo nuevas historias, en el ejercicio de reconstruir el pasado en el dialogar. Al decir de Margarita:

Un día alguien tendrá que escribir la historia de Sindihogar. Entonces como todas las historias y todas las luchas, no solo las de las mujeres, se van a quedar nombres, que son sumamente importantes, que hacen mucho y que no se reflejan. (Margarita, 2012, p. 4).

En su narración, se está contando, nos está contando a nosotras y denuncia con sus palabras, para que sus nombres y sus acciones se "re (actua) licen" en estos diálogos.

\section{Reflexiones finales}

En las narraciones de las trabajadoras encontramos un relato compartido, en cuanto a las carencias que se presentan dentro de la sociedad de acogida en sus procesos migratorios: estereotipos o marcas que influyen en sus relaciones laborales. Sin embargo, sus biografías son procesos singulares que interactúan con los marcos normativos preexistentes, subjetivándose. La necesidad de conseguir un ingreso económico y la facilidad que existe para emplearse en este tipo de trabajo, conlleva que en un mismo mercado de laboral se encuentren mujeres con trayectorias y modos de vida muy diversos.

Este universo heterogéneo se debe a la segmentación del mercado de trabajo por razones de género, clase y etnia. En éste, las mujeres, de forma situada las «mujeres inmigrantes», son relegadas a determinadas actividades y categorías profesionales con una fuerte demanda de trabajo hacia la reproducción social. El construirse como «mujer» dentro de estos espacios laborales, es producto de unas relaciones sociales que las preceden y que mantienen naturalizados el rol femenino del "cuidado", del "amor" y de la "entrega". Esta "mano invisible" a la que hace mención Cynthia (2012) culmina siendo el trabajo oculto y negado de las relaciones económicas entre los géneros que se encuentra asegurada por la división del trabajo y una organización social genérica (Lagarde, 1996). Así, los binarismo reproducen un reparto de tareas de forma generalizada y jerarquizada dentro del sistema patriarcal, donde las participantes narran sus dificultades para desmarcarse de estos lugares y poder pensarse de otra manera. Esto hace, que se internalice un modo de ser y estar para otros, donde se van desdibujando las referencias y necesidades de sí mismas.

Las relaciones de subordinación y jerarquización se hacen más evidentes en los relatos enmarcados dentro del trabajo puertas adentro (interno) que en los trabajos como externas. Disposición de tiempo libre, gestión del lugar, clase y género, son posiciones que marcan una diferencia en las valoraciones, percepciones y significados que hacen las participantes con relación a sus trabajos como internas y externas, desplegándose diferentes subjetividades respecto de los mismos.

Con relación a las implicaciones narradas en torno a la participación del sindicato, los recorridos también han sido singulares, atravesados por problemas estructurales que muchas veces condicionan el involucramiento.

En cuanto a los aspectos singulares, la pertenencia a este espacio es narrado desde los aprendizajes y experiencias que se comparten, junto a las dificultades en torno a subjetividades heterogéneas y su renegociación hacia un objetivo común (los derechos de las trabajadoras/es del hogar). El grado de participación en el sindicato también se encuentra relacionado al poder conciliar la vida laboral con la sindical, donde muchas veces se expresa el constreñimiento que implican las interminables horas de trabajo y las restricciones de salidas con mayor frecuencia en el caso de las trabajadoras internas.

No obstante, el tránsito por el sindicato participa en la resignificación de las experiencias de las participantes. El pasaje se realiza desde el relatar sus vivencias y que éstas se perpetúen en el ámbito discursivo, hacia emprender acciones que modifiquen de algún modo $\mathrm{u}$ otro sus situaciones laborales y personales. Así, nuevas 
prácticas performan escenarios políticos diversos. Estos hacen referencia a la organización de comisiones de trabajo, las concentraciones y manifestaciones realizadas, las entrevistas, tanto a los medios de comunicación como a otras entidades. Con ello, lo privado irrumpe en lo público, haciendo frente a la invisibilidad que trae aparejada el trabajo del hogar, constituyéndose modos de ser o subjetividades distintas.

El sindicato se presenta como un lugar propicio para construir un sujeto político, abriendo un campo de posibilidad para instalar debates y alianzas en torno a los trabajos del hogar y cuidados, como también, desde las activistas que protagonizan esta acción colectiva, para proclamar la demanda de derechos a condiciones laborales justas.

\section{Referencias bibliográficas}

Acharya, J. \& Lund, R. (2002). Gendered spaces: socio-spatial selations of self- employed women in craft production, orissa, India. Norsk geografisk tidsskrift- Norwegion Journal of geography $563,207-18$

Attanapola, C. (2005). Experiences of globalization and health in the narratives of women industrial workers in Sri Lanka. Gender technology and development, (9), 81-101

Álvarez, A. (2008). Habitando la frontera: empleadas domésticas procedentes de Rusia y ucrania. En: Castillo, S. \& Devillar, M. (Eds.) Tiempo de espera en las fronteras del mercado laboral: nuevos agentes sociales en el espacio social (pp. 33-48). Donostia: Ankulegi Antropologia Elkartea

Amigot, P. (2005). Relaciones de poder. Espacio subjetivo y prácticas de libertad: análisis genealógico de un proceso de transformación de género. Tesis Doctoral sin publicar. Departamento de Psicología Social. Facultad de Psicología. Universidad Autónoma de Barcelona

Balasch, M. \& Montenegro, M. (2003). Una propuesta metodológica desde la epistemología de los conocimientos situados: Las producciones narrativas. Encuentros En Psicología Social, 1(3), 44-48

Bermúdez, E. (2007). Historias de afecto y ternura, historias de desigualdad y discriminación: el discurso de mujeres inmigrantes internas en el servicio doméstico. Proyecto Equal: Analogías: historias vinculadas para la integración. Institut Universitari D`Estudis De La Dona Universitat De Valencia

Biglia, B. (2005). Narrativas de Mujeres Sobre Las Relaciones de género en los movimientos sociales. Tesis Doctoral no publicada. Departamento de Psicología Básica. Universidad de Barcelona.

Biglia, B. \& Bonet, J. (2009). La Construcción de narrativas como método de investigación psico-social. Prácticas de escritura compartida. Forum Qualitative Sozialforschung / Forum: Qualitative Social Research, 10(1).

Burman, E. (2000). Method, measurement, and madness. En: Holzman, L. \& Morss, J. (Eds.). Postmodern psychologies, societal practice, and political life (pp. 49-78). New York: Routledge

Butler, J. (1997). Lenguaje, poder e identidad. Madrid: Síntesis. 2004.

Carrasco, C. (1991). El trabajo doméstico. Un análisis económico. Madrid: Ministerio De Trabajo Y Seguridad Social. Colección Tesis Doctorales.
Carrasco, C. (2001). La sostenibilidad de la vida humana: ¿Un asunto de mujeres? Mientras Tanto, 81. Barcelona: Icaria.

Carrasco, C. (2006). La paradoja del cuidado: necesario pero invisible. Revista De Economía Crítica, 5, 39-64.

Castelló, L. (2008). La mercantilización y mundialización del trabajo reproductivo. El caso Español. Revista De Economía Crítica, 7, 74-94.

Catarino, C. \& Oso, L. (2000). La inmigración femenina en Madrid y Lisboa hacia una etnización del servicio doméstico y de las empresas de limpieza. Papers: Revista De Sociología, 60, 183-207.

Cynthia, 2012. Nosotras cuidamos, quién cuida de nosotras. Producción Narrativa. Manuscrito no publicado.

Colectivo IOE (2001). Mujer, inmigración y trabajo. Madrid: Instituto de Migraciones y Servicio Sociales, Ministerio de Trabajo y Asuntos Sociales.

Contreras, A. (2006). Derechos laborales de las trabajadoras domésticas nicaragüenses en Costa Rica. Managua (Nicaragua). Red nicaragüense de la sociedad civil para las migraciones. Cuaderno Migratorio 9, 53.

D’ Souza, A. (2010). Camino del trabajo decente para el personal del servicio doméstico: panorama de lo laboral. Suiza. Ed. Oit.

Escalera Karakola (2004). Diferentes diferencias y ciudadanías excluyentes. Una revisión feminista. En: AAVV. Otras inapropiables (pp. 9-33). Madrid: Traficantes de Sueños.

Ehrenreich, B. \& Hochschild, A. (2003). Global woman: Nannies, maids, and sex workers en the new economy. New York: Metropolitan Books

Femenías, M. (2003). Aproximación al pensamiento de Judith Butler. Comunicación presentada en la Conferencia de Gijón.

Foucault, Michel (1981). Tecnologías del yo. Barcelona: Paidós.

Foucault, M. (1984) Estética, ética y hermenéutica. Obras esenciales volumen III. Barcelona: Paidós.

Foucault, M. (1988). Sujeto y poder. Revista Mexicana de Sociología, $3(50), 3-20$

Foucualt, M. (1994). Hermenéutica del sujeto. Madrid: Ediciones La Piqueta.

Gadamer, H. (1975). Verdad y método. Salamanca: Ediciones Sígueme.

Gil, L. \& Orozco, A (2011). Desigualdades a flor de piel: cadenas globales de cuidados. Concreciones en el empleo de hogar y politicas públicas. Edición Onu Mujeres. Madrid: Creative Commons.

Gregorio, C. (2001). Mujeres inmigrantes ¿ciudadanas? En: De Prado, J. Diversidad cultural, identidad y ciudadanía (pp. 177197). Córdoba: Inet.

Gregorio, C. (2009). Mujeres inmigrantes: colonizando sus cuerpos mediante fronteras procreativas, étnico-culturales, sexuales y reproductivas. Viento Sur, 104, 42-54.

Gregorio, C. (2009a). Políticas de conciliación, externalización del trabajo doméstico de cuidados y migraciones transnacionales. Comunicación Economía Feminista, Baeza, abril de 2009.

Gregorio, C. (2010). Debates feministas en el análisis de la inmigración no comunitaria en el Estado Español. Reflexiones desde la etnografía y la antropología social. Relaciones Internacionales, 14, 93-115.

Haraway, D. (1991). Ciencia, cyborgs y mujeres. La reinvención de la naturaleza. Madrid: Ediciones Cátedra S.A (1995)

Isabel (2012). Los primero pasos. Producción Narrativa. Manuscrito no publicado.

Izquierdo, M. (2004). Del sexismo y la mercantilización del cuidado a su socialización: hacia una política democrática del cuidado. Congreso Internacional Sare 2003. Cuidar Cuesta: Costes Y Beneficios Del Cuidado. Emakunde, 1-39.

Juliano, D. (2001). El juego de las astucias. Mujer y construcción de modelos sociales alternativos. Madrid: Horas \& Horas. 
Lagarde, M. (1996). Género y feminismo. Desarrollo humano y democracia. Cuadernos Inacabados 25. Madrid: Horas \& Horas.

Larrañaga, M. \& Jubeto, Y. (2009). Los trabajos de cuidados remunerados: una mirada desde la comunidad Autónoma de Euskadi. Comunicación Presentada En III Congreso De Economía Feminista, Baeza.

Lerussi, R. (2008). Trabajadoras domésticas nicaragüenses en Costa Rica. En las encrucijadas de un debate feminista. Tesis Magíster no publicada. Universidad Autónoma De Barcelona.

Lund, R. (2000). Geographies of eviction, expulsion and marginalization: stories and coping strategies of The Veddhas, Sri Lanka. Norsk Geografisk Tidsskrift- Norwegian Journal of Geography 54,(3), 102-109.

Margarita (2012). Si todas viéramos el mismo color éste mundo no tendría sabor. Producción Narrativa. Manuscrito no publicado.

Martínez-Guzmán, A. (2009). Movimientos adyacentes para repensar la Investigación-Acción Participante. Disponible en: http:// psicologiasocial.uab.es/fic/ca/book/2009/02/16

Montenegro, M., Galaz, C., Yufra, L. \& Montenegro, K. (2011). Dinámicas de subjetivación y diferenciación en servicios sociales para Mujeres inmigradas en la ciudad de Barcelona. Athenea Digital, 11(2), 113-132. Disponible en: http://psicologiasocial. uab.es/athenea/index.php/atheneaDigital/article/view/709/574
Nicolás, G. (2009). Los trabajos invisibles: reflexiones feministas sobre el trabajo de las Mujeres. Repensar l'economia i els treballs de l'experiència de les dones. Comunicación Presentada En Las Jornadas Organizadas Por Surt, Barcelona.

Parella, S. (2002). La internacionalización de la reproducción. La inserción laboral de la mujer inmigrante en los servicios de proximidad. Tesis Doctoral no publicada. Departamento de Sociología. Universidad Autónoma de Barcelona. Disponible en: http://www. tdx.cat/handle/10803/5110

Parreñas, S. (2001). Servants of globalization. Women, migration, and domestic work. Stanford: Stanford University Press

Pérez, A. (2006). Perspectivas feministas en torno a la economía: el caso de Los cuidados. Madrid: Consejo Económico y Social.

Ramona (2012). Dejando una pequeña mancha. Producción Narrativa. Manuscrito no publicado.

Riessman, C. (2002). Analysis of personal narrative. En: Gubrium, J. \& Holstein, J. (Eds.). Handbook of interview research: context and method. London: Sage.

Sipi, R. (2000). Las asociaciones de mujeres ¿agentes de Integración social? Papers: Revista De Sociología, 60, 355-364.

Vandsemb, B. (1995). The place of narrative in the study of third world migration: the case of spontaneous rural migration In Sri Lanka. Professional Geographer, 47(4), 411-25. 